\title{
On Dynamics of Brans-Dicke Theory of Gravitation
}

\author{
Hossein Farajollahi* \\ Department of Physics, University of Guilan, Rasht, Iran \\ Mehrdad Farhoudit and Hossein Shojait $\ddagger$ \\ Department of Physics, Shahid Beheshti University, G.C., Evin, Tehran 19839, Iran
}

(Dated: November 3, 2018)

\begin{abstract}
We study longstanding problem of cosmological clock in the context of Brans-Dicke theory of gravitation. We present the Hamiltonian formulation of the theory for a class of spatially homogenous cosmological models. Then, we show that formulation of the Brans-Dicke theory in the Einstein frame allows how an identification of an appropriate cosmological time variable, as a function of the scalar field in the theory, can be emerged in quantum cosmology. The classical and quantum results are applied to the Friedmann-Robertson-Walker cosmological models.
\end{abstract}

PACS numbers: 04.20.Cv; 04.50.-h; 04.60.Ds; 98.80.Qc

Keywords: Cosmological Time; Brans-Dicke Theory of Gravitation; Einstein Frame; Quantum Cosmology.

*Electronic address: hosseinf@guilan.ac.ir

${ }^{\dagger}$ Electronic address: m-farhoudi@sbu.ac.ir

${ }^{\ddagger}$ Electronic address: h-shojaie@sbu.ac.ir 


\section{INTRODUCTION}

Identifying a suitable cosmological clock with respect to which the dynamics of the Universe can be measured is one of the most fundamental problems in quantum cosmology. The conventional Wheeler-DeWitt (WD) formulation gives a time-independent quantum theory, and consequently, observers experience the passage of time. Although the problem manifests itself in quantum level, it is originated from classical theories of gravity, in which the theory is invariant under time re-parametrization, see, e.g., Refs. [1-3] and references therein.

In the context of general relativity, a lot of efforts have been directed to solve the problem via re-interpretation of time notion, or introducing a material time variable emerged from the geometry or matter of four dimensional space-time, and or introduction of any other kind of geometrical objects [4-17]. In particular, introduction of a scalar field in the time reparametrization invariant theories, e.g. general relativity, to present a cosmological clock has been investigated [18 25]. Unfortunately, there still does not exist a completely satisfactory solution to this fundamental issue.

On the other hand, physicists have long entertained the scalar-tensor theories of gravitation as alternative ones and the most popular rivals to the general relativity, in which the nature simplest imaginable phenomenon, namely a scalar field, plays the major role. The scalar-tensor theory, developed originally by Jordan, began by embedding a four dimensional curved manifold in five dimensional flat space-time [26].

The Brans-Dicke gravitational theory is the well-known example of the scalar-tensor theories, in which the gravitational interaction, besides the usual metric of general relativity, is mediated by a scalar field, which has the physical effect of changing the effective gravitational constant from place to place. According to the Brans-Dicke assumption [27], the scalar field decoupled from the matter part of the Lagrangian in order to save the weak equivalence principle being violated. However, this assumption hardly seems to be supported by any example of more fundamental theories and alternatively, it has been shown that the size of a compactified internal space can behave as a four dimensional scalar field of the nature of the Brans-Dicke model, with a parameter determined uniquely in terms of the dimensionality of space-time [28].

In the cosmological context, scalar fields have been supposed to cause an accelerating 
expanded universe [29], to solve the horizon problem and to give a hypothetical reason for the non-vanishing cosmological constant. Massless, or long-ranged, scalar fields, in this context, are known as inflatons and massive, or short-ranged, scalar fields are proposed to use, for example, Higgs-like fields [30]. In this work, we purpose to identify another role for the scalar field in the Brans-Dicke theory in order to solve the problem of time in quantum cosmology. Since the usual time coordinate in four dimensional space-time is not a suitable candidate to play the role of cosmological time, one may speculate that the dynamical Brans-Dicke scalar field - decoupled from matter in the Lagrangian or originated from higher dimensional space - is eligible to perform such a role similar to the role of Newtonian external time parameter in the classical mechanics.

The non-minimal coupling term in the scalar-tensor theory is equivalent to the presence of conformal transformations. That is, one can change the form of this coupling term by applying these transformations. Among these transformations, the ones which can transform the coupling term into a constant are more attractive, for they can be employed to switch between the Jordan and Einstein frames, which are the most discussed conformal frames [31, 32]. Some aspects of the cosmological conformal equivalence between these two frames have also been investigated [22]. Nonetheless, the physics is not invariant under conformal transformations, except in the weak gravitational field limit, and one should choose one of them as the physical frame. As the physical frame labels the frame we live in, it can be selected upon physical grounds consistent with principles and observations.

In this manuscript, in section two, we start with the description of the Brans-Dicke theory and present it in the Einstein frame. The advantage of Einstein frame over the Jordan frame is that the scalar field contribution to the Brans-Dicke Lagrangian density is identical to one used in the vacuum general relativity, where it gives the possibility of emerging a cosmological time variable in the theory as we will discuss in this work. In section three, the Hamiltonian formulation of the theory is presented. The formulation is given for spatially homogenous cosmological models which provide good prototypes for many theoretical models in gravity and cosmology. Then, a cosmological time variable is introduced, as a function of the scalar field in the theory, in which the dynamics of the metric functions can be performed. In section four, as an example, the classical and quantum dynamics of the Friedmann-Robertson-Walker (FRW) models are studied. Finally, section five presents conclusions and remarks drawn from this work. 


\section{BRANS-DICKE THEORY OF GRAVITATION IN EINSTEIN FRAME}

The Brans-Dicke theory of gravitation, based on the Mach principles, is presented by a general action for a real scalar field living in a four dimensional curved space-time with the (physical) Lagrangian density given by

$$
\mathcal{L}_{\mathrm{BD}}=\sqrt{-g}\left(\varphi R-\frac{\omega}{\varphi} g^{\mu \nu} \partial_{\mu} \varphi \partial_{\nu} \varphi\right)
$$

where the lower Greek indices run from zero to three, $R$ is the Ricci curvature scalar formed from the metric $g_{\mu \nu}$. The positive value $\varphi\left(x^{\rho}\right)$ is called Jordan's scalar field and the dimensionless constant $\omega$ is the only parameter of theory. We assume that there is no other matter field in the theory. The $\varphi R$ term is a non-minimal coupling term, in which $G^{-1}$ in the Einstein-Hilbert term is replaced with $\varphi$. This provides that, as long as the scalar field varies slowly, the Universe is assumed to feel an effective spatially uniform gravitational constant $G_{\text {eff }}=1 / \varphi$ which depends only on the cosmic time. The second term is proportional to the kinetic term of the scalar field. To remove the singular behavior of $\varphi^{-1}$ from

this term, one can replace it by introducing a new real scalar field, as $\phi=\sqrt{8|\omega| \varphi}$, that converts (11) to

$$
\mathcal{L}_{\mathrm{BD}}=\sqrt{-g}\left(\frac{1}{8|\omega|} \phi^{2} R-\frac{1}{2} \epsilon g^{\mu \nu} \partial_{\mu} \phi \partial_{\nu} \phi\right)
$$

where $\epsilon= \pm 1$ keeps the sign of $\omega$. It is worth noting that when $\omega<0$, the scalar field can be interpreted as a ghost [28].

The Lagrangian density (2) has been written in the Jordan frame as a physical frame, however we intend to proceed in the Einstein frame. This can be performed by employing a conformal transformation such that it transforms the coefficient of $R$ to a constant, where the causality structure does not change. Mathematically, the conformal transformation $\widehat{g}_{\mu \nu}=\Omega^{2}\left(x^{\rho}\right) g_{\mu \nu}$ transforms (2) to

$$
\mathcal{L}_{\mathrm{BD}}=\sqrt{-\widehat{g}} \Omega^{-2}\left\{\frac{1}{8|\omega|} \phi^{2}\left[\widehat{R}+6 \widehat{\square} \ln \Omega+6 \widehat{g}^{\mu \nu} \partial_{\mu}(\ln \Omega) \partial_{\nu}(\ln \Omega)\right]-\frac{1}{2} \epsilon \widehat{g}^{\mu \nu} \partial_{\mu} \phi \partial_{\nu} \phi\right\} .
$$

Choosing $\Omega=\phi / \sqrt{8|\omega|}$ and eliminating the second term of the Lagrangian density by integrating by parts, it yields

$$
\mathcal{L}_{\mathrm{BD}}=\sqrt{-\widehat{g}}\left(\widehat{R}-\frac{1}{2} \lambda \widehat{g}^{\mu \nu} \partial_{\mu} \phi \partial_{\nu} \phi\right)
$$


where

$$
\lambda \equiv \Omega^{-2}\left[\epsilon+12\left(\frac{d \Omega}{d \phi}\right)^{2}\right]=\frac{8}{\phi^{2}}\left(\epsilon|\omega|+\frac{3}{2}\right) .
$$

For $\phi$ to be a normal field instead of a ghost, $\lambda$ is positive when $\omega>-3 / 2$. Actually, this restriction depends on the dimension of space-time $D$, namely $\omega>-(D-1) /(D-2)$ [33, 34].

Now, one can introduce another new scalar field, by $\partial_{\mu} \psi=\sqrt{\lambda} \partial_{\mu} \phi$, and finally rewrites (4) as

$$
\mathcal{L}_{\mathrm{BD}}=\sqrt{-\widehat{g}}\left(\widehat{R}-\frac{1}{2} \widehat{g}^{\mu \nu} \partial_{\mu} \psi \partial_{\nu} \psi\right),
$$

which is obviously the Lagrangian density in the Einstein frame with a canonical kinetic term.

The Lagrangian density (6) is actually the well-known Lagrangian of vacuum general relativity plus contribution from a scalar field. However, one usually lacks physical justifications to priori introduce such a kinetic term when working with vacuum general relativity, although via the induced-matter theory [35, 36], one may be able to get such an effective induced kinetic term. Nevertheless, through this work and by introducing a cosmological time, we will also offer a physical base to justify starting with the Lagrangian density (6) right at the beginning.

\section{HAMILTONIAN FORMULATION AND COSMOLOGICAL TIME}

While in the present work, for its relative simplicity, a Lagrangian formulation of the theory is given, we are more interested in the Hamiltonian version of the theory where the absence of time displays itself in a freezing Hamiltonian. For this purpose, we consider the Hamiltonian formulation of the theory.

Suppose that the four dimensional space-time manifold can be foliated by homogeneous, but generally anisotropic, spatial hypersurfaces $\Sigma_{t}$ of constant time t. Then, the associated space-time metric can have the form

$$
d s^{2}=-N^{2}(t) d t^{2}+\widehat{g}_{i j} d x^{i} d x^{j}=-N^{2}(t) d t^{2}+a^{2}(t) \gamma_{i j} d x^{i} d x^{j}
$$

where the lower Latin indices run from one to three, $N(t)$ is a lapse function and $\gamma_{i j}$ is the time-independent spatial 3-metric on the hypersurfaces $\Sigma_{t}$. For convenience, we assume that the time axis is always normal to the hypersurfaces of homogeneity $\Sigma_{t}$. We also restrict the scalar field (dilaton) to be spatially homogeneous. 
From Lagrangian (6) and metric (7), the canonical momentum associated with the dynamical variable $\psi$ is

$$
\pi_{\psi}=\sqrt{(3) \widehat{g}} N^{-1} \dot{\psi}
$$

where ${ }^{(3)} \widehat{g}=a^{6}(t) \gamma$ is the determinant of $3-$ metric on $\Sigma_{t}$ and $\gamma$ is the determinant of $\gamma_{i j}$. Following the Dirac procedure [37], the total Hamiltonian obtained from the action, by a Legendre transformation, is

$$
H_{\text {tot }}=\int_{\Sigma_{t}} N\left(\mathcal{H}+\frac{\pi_{\psi}^{2}}{2 \sqrt{(3) \widehat{g}}}\right) d^{3} x .
$$

The variable $N$ plays the role of a Lagrange multiplier and $\mathcal{H}$ is the super-Hamiltonian, generally, given by

$$
\mathcal{H}\left(t, x^{i} ; \widehat{g}_{i j}, \pi^{i j}\right)=\mathcal{G}_{m n k l}\left(t, x^{i}\right) \pi^{m n}\left(t, x^{i}\right) \pi^{k l}\left(t, x^{i}\right)-\sqrt{{ }^{(3)} \widehat{g}} R\left(t, x^{i}, \widehat{g}_{i j}\right),
$$

in which

$$
\mathcal{G}_{m n k l}\left(t, x^{i}\right)=\frac{1}{2 \sqrt{(3) \widehat{g}}}\left[\widehat{g}_{m k}\left(t, x^{i}\right) \widehat{g}_{n l}\left(t, x^{i}\right)+\widehat{g}_{n k}\left(t, x^{i}\right) \widehat{g}_{m l}\left(t, x^{i}\right)-\widehat{g}_{m n}\left(t, x^{i}\right) \widehat{g}_{k l}\left(t, x^{i}\right)\right]
$$

is the DeWitt supermetric on the space of $3-$ metrics and $\pi^{i j}$ is the conjugate momentum of $\widehat{g}_{i j}$. We thus have a new first class Hamiltonian constraint,

$$
\mathcal{H}+\frac{\pi_{\psi}^{2}}{2 \sqrt{{ }^{(3)} \widehat{g}}} \approx 0
$$

instead of $\mathcal{H} \approx 0$. By the non-degenerate character of the metric, the new Hamiltonian constraint can be redefined by rescaling $N$ as $N_{*}=N / a^{3}$. Namely

$$
\mathcal{H}_{*}=a^{3} \mathcal{H}+\frac{\pi_{\psi}^{2}}{2 \sqrt{\gamma}} \approx 0,
$$

where the total Hamiltonian then becomes

$$
H_{\mathrm{tot}}=\int_{\Sigma_{t}} N_{*} \mathcal{H}_{*} d^{3} x
$$

In the Hamiltonian formulation of the theory, one can perform a canonical transformation from $\left(\psi, \pi_{\psi}\right)$ to $\left(T, \Pi_{T}\right)$ that preserves the form of equations of motion. Choosing the new canonical coordinate as

$$
T(t)=\frac{\psi}{\pi_{\psi}} \gamma
$$


then by calculating the Dirac bracket relation, one finds that

$$
\Pi_{T}=\frac{\pi_{\psi}^{2}}{2 \gamma}
$$

An analogy with unimodular general relativity [38-40] arises an anticipation that this new variable $T(t)$ may play the role of a cosmological time variable. This hope is fulfilled. Actually, the equation of motion derived from (15) implies that

$$
\frac{d T}{d t}=\left[T, H_{\text {tot }}\right]=\int_{\Sigma_{t}} N_{*} \sqrt{\gamma} d^{3} x
$$

and hence $N_{*} \sqrt{\gamma}$ presents the density of $\dot{T}$ over the hypersurface. By integrating, one obtains

$$
T(t)=\int N_{*}{ }^{(3)} V_{\text {proper }} d t
$$

Therefore, $T(t)$ is just the 4 -volume between the hypersurfaces $\Sigma_{t_{0}}$ and $\Sigma_{t}$ with $t_{0}$ as an initial time, and equation (17) shows that the rate of change of this time variable is necessarily a positive value.

In the Einstein frame formulation of the Brans-Dicke cosmology, $T(t)$ coincides classically with the cosmological time parameter which arises both in the unimodular general relativity [38 41] and in Sorkin's sum over histories approach [42]. Note that, $T(t)$ is a monotonically increasing function along any future directed time-like curve, and thus can indeed be used to parameterize this trajectory.

The gauge invariant quantity $T(t)$ is not a Dirac observable, for it does not commute with $\mathcal{H}_{*}$, but its conjugate momentum, $\Pi_{T}$, is a Dirac observable. Besides, $T(t)$ can be used as a clock. Indeed, if we label the spatial hypersurfaces by the cosmological time $T(t)$ instead of the coordinate time $t$, and foliate the space-time by $T(t)$ and $\Sigma_{T}$ rather than $t$ and $\Sigma_{t}$, then any geometric, i.e. generally covariant, quantity defined on $\Sigma_{T}$ has vanishing Poisson bracket with the integrated Hamiltonian constraint.

Note that, in the formulation of the theory in terms of the old canonical variables, $\left(\psi, \pi_{\psi}\right)$, in quantizing the theory one obtains a Klein-Gordon like equation and needs to perform a decomposition of the solution in order to get a Schrödinger-like equation. However, in terms of the new canonical variables, $\left(T, \Pi_{T}\right)$, we directly acquire a Schrödinger-like equation. Indeed, in the quantum theory, the momentum variable $\Pi_{T}$ is represented by the operator $-i \partial / \partial T$ and the WD equation of the Hamiltonian constraint (13) takes the form 
of a Schrödinger equation describing the evolution of cosmological wave functions, e.g. $\Psi$, with respect to the cosmological time parameter, $T(t)$, as

$$
i \frac{\partial \Psi}{\partial T}=\frac{a^{3}}{\sqrt{\gamma}} \mathcal{H} \Psi
$$

In order to realize properties and effects of such a cosmological time, we probe the dynamical behaviour of the universe in the FRW models with respect to this clock in the next section.

\section{THE FRW MODELS}

As an example, we begin with the line element for the FRW models in the spherical coordinates, namely metric (7) in which $a(t)$ is the cosmic scale factor - that determines the radius of universe - and $\gamma_{i j}$ is the time-independent metric of the three-dimensional maximally symmetric spatial sections

$$
\gamma_{i j} d x^{i} d x^{j}=\frac{d r^{2}}{1-k r^{2}}+r^{2}\left(d \theta^{2}+\sin ^{2} \theta d \phi^{2}\right)
$$

with the constant curvature scalar ${ }^{(3)} R\left(\gamma_{i j}\right)=6 k$.

The Brans-Dicke action in the Einstein frame for the FRW models with a minimally coupled massless scalar field to gravity is given by

$$
S\left[g_{\mu \nu}, \psi\right]=\int\left[6\left(-\frac{a \dot{a}^{2}}{N}+k N a\right)+\frac{a^{3}}{2 N} \dot{\psi}^{2}\right] \sqrt{\gamma} d^{4} x .
$$

Hence, the total Hamiltonian is

$$
H_{\mathrm{tot}}=\int_{\Sigma_{t}} N\left(-\frac{p_{a}^{2}}{24 a \sqrt{\gamma}}-6 k a \sqrt{\gamma}+\frac{\pi_{\psi}^{2}}{2 a^{3} \sqrt{\gamma}}\right) d^{3} x .
$$

Now, one can write the total Hamiltonian as

$$
H_{\mathrm{tot}}=\int_{\Sigma_{t}} N_{*}\left(-\frac{a^{2} p_{a}^{2}}{24 \sqrt{\gamma}}-6 k a^{4} \sqrt{\gamma}+\Pi_{T} \sqrt{\gamma}\right) d^{3} x
$$

where again we apply $N_{*}=N / a^{3}$ as a rescaling. Following the procedure in the previous section, the first class constraint can be redefined as

$$
0 \approx \mathcal{H}_{*}=-\frac{a^{2} p_{a}^{2}}{24 \sqrt{\gamma}}-6 k a^{4} \sqrt{\gamma}+\Pi_{T} \sqrt{\gamma}
$$


The classical equations can be obtained from (23) as

$$
\begin{gathered}
\dot{a}=\frac{\partial\left(N_{*} \mathcal{H}_{*}\right)}{\partial p_{a}}=-\frac{N_{*} a^{2} p_{a}}{12 \sqrt{\gamma}} \\
\dot{p}_{a}=-\frac{\partial\left(N_{*} \mathcal{H}_{*}\right)}{\partial a}=N_{*}\left(\frac{a p_{a}^{2}}{12 \sqrt{\gamma}}+24 k a^{3} \sqrt{\gamma}\right), \\
\dot{T}_{\mathrm{pd}}=\frac{\partial\left(N_{*} \mathcal{H}_{*}\right)}{\partial \Pi_{T}}=N_{*} \sqrt{\gamma},
\end{gathered}
$$

and

$$
\dot{\Pi}_{T}=-\frac{\partial\left(N_{*} \mathcal{H}_{*}\right)}{\partial T}=0
$$

where $\dot{T}_{\mathrm{pd}}$ is the proper density of cosmological time. Equation (28) shows that $\Pi_{T}$ is a constant of motion. By relation (8), one gets $\dot{T}_{\mathrm{pd}}=\gamma \dot{\psi}$ which simply shows that the dynamics of the cosmological time variable, $T$, is proportional to the dynamics of the scalar field.

We are more interested to employ the cosmological time, $T(t)$, and find the behavior of the isotropic variables $a$ and $p_{a}$ with respect to it. Thus, we find

$$
a^{\prime}=-\frac{a^{2} p_{a}}{12 \gamma}
$$

and

$$
p_{a}^{\prime}=\frac{a p_{a}^{2}}{12 \gamma}+24 k a^{3}
$$

where the prime denotes derivative with respect to $T$. In the following, we investigate the behavior of scale factor with respect to the cosmological time $T$ for various curvatures.

Case $\mathrm{k}=0$ :

Solving the last two equations (29) and (30), assuming $k=0$, gives

$$
a(T)=a_{0} e^{-C T}
$$

and

$$
p_{a}(T)=p_{a_{0}} e^{C T}
$$

where $a_{0}=\left.a\right|_{T=0}, p_{a_{0}}=\left.p_{a}\right|_{T=0}$ and $C \equiv a(T) p_{a}(T) / 12$ is a constant. A negative $C$ with positive $a_{0}$ provides an accelerating expanded universe, with a positive constant Hubble, $H=-C$. In this case, universe expands exponentially according to the cosmological time variable, and naturally accelerating without a beginning singularity. 
Indeed, the monotonic dependence of the dynamical clock $T$ with respect to the scale factor shows that it can be employed as a cosmological time for the gravitational dynamics.

\section{Case $\mathrm{k}=1$ :}

The nontrivial solution of the above coupled non linear differential equations (29) and (30), for the scale factor gives

$$
a(T)=\frac{\sqrt{2 \sqrt{c_{1}} c_{2}} e^{\sqrt{c_{1}} T}}{\gamma^{1 / 4}\left(1+c_{2}^{2} e^{4 \sqrt{c_{1}} T}\right)^{1 / 2}},
$$

where $c_{1}$ and $c_{2}$ are positive constants of integrations. This solution shows that universe has no singularity at all and for small enough $T$ the scale factor also becomes very small. The solution goes to zero when $T$ goes to minus infinity. Besides, universe shrinks to a big crunch as $T$ goes to infinity while it reaches a maximum size during its history.

Case $\mathrm{k}=-1$ :

In this case the solution is

$$
a(T)=\frac{\sqrt{2 \sqrt{c_{3}} c_{4}} e^{\sqrt{c_{3}} T}}{\gamma^{1 / 4}\left(1-c_{4}^{2} e^{4 \sqrt{c_{3}} T}\right)^{1 / 2}},
$$

where again $c$ 's are positive constants of integrations. This solution shows that the scale factor goes to infinity for some $T$, depends on the constants of integrations, and becomes imaginary beyond it. Such a scale factor does not have a well physical interpretation.

When the system is canonically quantized, the associated WD equation, i.e. the Schrödinger-like equation, describes how the wave function of universe evolves with the cosmological time variable. From equation (24), we have

$$
-\partial_{a}^{2} \Psi+144 k \gamma a^{2} \Psi+i \frac{24 \gamma}{a^{2}} \partial_{T} \Psi=0,
$$

where for wave function $\Psi(a, T)$ as

$$
\Psi_{E}(a, T)=\Psi(a) e^{i E T},
$$

$\Psi(a)$ satisfies

$$
-\frac{a^{2}}{24 \gamma} \partial_{a}^{2} \Psi(a)+\left(6 k a^{4}\right) \Psi(a)=E \Psi(a) .
$$


In order to have a self-adjoint Hamiltonian, the inner product between wave functions must be

$$
<\Phi, \Psi>(T)=\int_{0}^{\infty} a^{-2} \Phi^{*} \Psi d a,
$$

and solutions must satisfy the convenient boundary conditions

$$
\Psi(0, T)=0 \quad \text { or }\left.\quad \frac{\partial \Psi(a, T)}{\partial a}\right|_{a=0}=0,
$$

in the domain of Hamiltonian operator [43, 44].

In the following, we find cosmological wave functions for various curvatures.

Case $\mathrm{k}=0$ :

For spatially flat space-time, equation (37) is an Euler-type equation

$$
\partial_{a}^{2} \Psi(a)+24 \gamma a^{-2} E \Psi(a)=0
$$

and its general solution is

$$
\Psi_{E}(a, T)=e^{i E T} \sqrt{a}\left(c_{5} a^{\frac{\sqrt{1-96 \gamma E}}{2}}+c_{6} a^{-\frac{\sqrt{1-96 \gamma E}}{2}}\right)
$$

where c's are constants of integrations. Obviously, the above solution is not square integrable and in order to obtain a possible physical solution, one should construct wave packets as

$$
\Psi(a, T)=\int_{0}^{\infty} A(E) \Psi_{E}(a, T) d E .
$$

However, even with the above wave packets, still the ill-behavior of solution (41) prevents one to get finite-norm states by superposing them.

\section{Case $\mathrm{k}=1$ :}

The solution for positive curvature is

$$
\Psi_{E}(a, T)=e^{i E T} \sqrt{a}\left[c_{7} K_{\nu}\left(6 \gamma a^{2}\right)+c_{8} I_{\nu}\left(6 \gamma a^{2}\right)\right]
$$

where again c's are constants of integrations, $K_{\nu}$ and $I_{\nu}$ are the modified Bessel functions and $\nu=\sqrt{1-96 \gamma E} / 4$. Since $I_{\nu}$ grows exponentially as a goes to infinity, one must set $c_{8}=0$, and consequently the first boundary condition (39) is satisfied. However, it is not easy to find an explicit finite-norm solution to the WD equation by superposing stationary states, for the integrals over the order of modified Bessel functions are not easy to perform. 
Case $\mathrm{k}=-1$ :

The solution for negative curvature is

$$
\Psi_{E}(a, T)=e^{i E T} \sqrt{a}\left[c_{9} J_{\mu}\left(6 \gamma a^{2}\right)+c_{10} Y_{\mu}\left(6 \gamma a^{2}\right)\right]
$$

where $c$ 's are constants of integrations, $J_{\mu}$ and $Y_{\mu}$ are the Bessel functions and $\mu=$ $\sqrt{1+96 \gamma E} / 4$. If $0<\gamma E<1 / 96$ both boundary conditions (39) can be fulfilled, but an explicit wave packet cannot be found by superposition of stationary states, for very few results are known for integrals over the order of Bessel functions.

Note that, the above equations for $k=0, \pm 1$ are similar to the WD equations obtained for perfect fluid cosmological models with stiff matter in Ref. [45].

\section{CONCLUSIONS AND REMARKS}

The problem in general theory of relativity goes against the simple Newtonian picture of the fixed, absolute and external time parameter. The classical theory, while itself free from problems relating to the definition and interpretation of time, contains indications of problems in the quantum theory, where the absence of a time parameter is hard to reconcile with our everyday experience. In particular, one of the most fundamental questions in quantum cosmology, that of identifying a suitable time parameter with respect to which the dynamics of the Universe can be measured, is unsolved. Alternatively, in this article, in the Brans-Dicke theory, we address the promising possibility of the scalar field, that may be originated from extra dimension in the theory, to resemble the Newtonian external time parameter. This can be of interest for those physicists who believe that only an "external" time parameter, same as the one we experience in the Newtonian classical mechanics, can really solve the problem of time.

In this work, we first formulate the Brans-Dicke theory of gravitation in the Einstein frame, in which it is identical to general relativity with a contribution from a scalar field. Then, we present the Hamiltonian formulation of spatially homogenous cosmological model for the theory. Due to the presence of the minimally coupled scalar field term in the formulation, we show that the dynamics of the metric functions can be obtained using a time variable, $T(t)$, as a function of the scalar field. Even though, the time variable is not a Dirac observable, it can be used to label spatial hypersurfaces and plays the role of a cosmological 
clock. Besides, the conjugate momentum to this cosmological clock is a Dirac observable. It has to be emphasized that the derived dynamical time, with its physical significant, is emerged from the formulation of the theory in the Einstein frame. This can be considered as an advantage of this frame over the Jordan frame in which the Brans-Dicke theory has been written. Indeed, the introduced cosmological time may justify the Einstein frame with the Lagrangian of vacuum general relativity plus a kinetic term of a scalar field as a physical frame.

We finally apply the results for the classical and quantum FRW models. We find that the classical models have solutions which avoid the usual initial cosmological singularity. Particularly, in a positive curvature space-time, the solution also shows a big crunch in future while reaches a turning point during its evolution. In the quantum description of the FRW models, though it is hard to obtain a physical solution via canonical quantization due to difficulty in solving the spatial part of the WD equation, the wave function of universe depends on the dynamical variable $T$.

[1] R. Benedetti and E. Guadagnini, Nucl. Phys. B 613, 330 (2001).

[2] C. Kiefer, Quantum Gravity, (Oxford University Press, Oxford, 2007).

[3] R. Gambini, R.A. Porto, J. Pullin and S. Torterolo, Phys. Rev. D 79, 041501R (2009).

[4] C. Isham and K. Kuchar, Ann. Phys. 164, 288 (1985).

[5] K. Kuchar, Time and Interpretations of Quantum Gravity, in Proc. $4^{\text {th }}$ Canadian Conference on General Relativity and Relativistic Astrophysics, Ed. G. Kunstatter, D. Vincent and J. Williams, (World Scientific, Singapore, 1992), pp. 211.

[6] R.M. Wald, Phys. Rev. D 48, R2377 (1993).

[7] C.G. Torre, "The problem of time and observables: some recent mathematical results", gr-qc/9404029.

[8] F.H. Gaioli and E.T.G. Alvarez, Gen. Rel. Grav. 26, 1267 (1994).

[9] A. Higuchi and R.M. Wald, Phys. Rev. D 51, 544 (1995).

[10] J. Feinberg and Y. Peleg, Phys. Rev. D 52, 1988 (1995).

[11] R. De Pietri and C. Rovelli, Class. Quant. Grav. 12, 1279 (1995).

[12] N. Grot, C. Rovelli and R.S. Tate, Phys. Rev. A 54, 4676 (1996). 
[13] S. Kauffman and L. Smolin, "A possible solution to the problem of time in quantum cosmology", gr-qc/9703026.

[14] G. Landi and C. Rovelli, Phys. Rev. Lett. 78, 3051 (1997).

[15] J.J. Halliwell, The Future of Theoretical Physics and Cosmology, in Proc. of S. Hawking's 60 ${ }^{\text {th }}$ Birthday Conference, Cambridge, U.K., 2002, pp. 675.

[16] C. Rovelli, Phys. Rev. D 65, 044017 (2002).

[17] C. Rovelli, Phys. Rev. D 65, 124013 (2002).

[18] C.J. Isham, "Canonical quantum gravity and the problem of time", gr-qc/9210011.

[19] P. Hajicek, Class. Quant. Grav. 13, 1353 (1996).

[20] T. Thiemann, "Solving the problem of time in general relativity and cosmology with phantoms and k-essence", astro-ph/0607380.

[21] P. Hajicek, Nucl. Phys. Proc. Suppl. 57, 11 (1997).

[22] S. Capozziello, R. de Ritis and A.A. Marino, Class. Quant. Grav. 14, 3243 (1997).

[23] T.P. Shestakova1 and C. Simeone, Grav. Cosmol. 10, 161 (2004).

[24] H. Farajollahi, Int. J. Theor. Phys. 47, 6 (2008)

[25] H. Farajollahi and A. Ravanpak, Int. J. Theor. Phys. 48, 12 (2009)

[26] P. Jordan, Schwerkraft und Weltall, (Friedrich Vieweg and Son, Braunschweig, 1955).

[27] C. Brans and R.H. Dicke, Phys. Rev. 124, 925 (1961).

[28] Y. Fujii and K. Maeda, The Scalar-Tensor Theory of Gravitation, (Cambridge University Press, Cambridge, 2004).

[29] A.H. Guth, Phys. Rev. D 23, 347 (1981).

[30] J.L. Cervantes-Cota and H. Dehnen, Phys. Rev. D 51, 395 (1995).

[31] V. Faraoni and E. Gunzig, Int. J. Theor. Phys 38, 217 (1999).

[32] S. Capozziello, P. Martin-Moruno c and C. Rubano, Phys. Let. B 689, 117 (2010).

[33] J.D. Barrow, D. Kimberly and J. Magueijo, Class. Quant. Grav. 21, 4289 (2004).

[34] M.P. Dabrowski, T. Denkiewicz and D. Blaschke, Ann. Phys. 16, 237 (2007).

[35] P.S. Wesson, Space-Time-Matter, (World Scientific, Singapore, 1999).

[36] P.S. Wesson, Five-Dimensional Physics, (World Scientific, Singapore, 2006).

[37] P.A.M. Dirac, Proc. Roy. Soc. A 165, 199 (1938).

[38] W.G. Unruh, Phys. Rev. D 40, 1048 (1989).

[39] M. Henneaux and C. Teitelboim, Phys. Lett. B 222, 195 (1989). 
[40] W.G. Unruh and R.M. Wald, Phys. Rev. D 40, 2598 (1989).

[41] H. Farajollahi, Gen. Rel. Grav. 37, 383 (2005).

[42] R.D. Sorkin, Int. J. Theor. Phys. 33, 523 (1991).

[43] N.A. Lemos, J. Math. Phys. 37, 1449 (1996).

[44] F.G. Alvarenga and N.A. Lemos, Gen. Rel. Grav. 30, 681 (1998).

[45] F.G. Alvarenga, J.C. Fabris, N.A. Lemos and G.A. Monerat, Gen. Rel. Grav. 34, 651 (2002). 